\title{
Towards Region-based Memory Management for Mercury Programs *
}

\author{
Quan Phan and Gerda Janssens \\ Department of Computer Science, K.U.Leuven \\ Celestijnenlaan, 200A, B-3001 Heverlee, Belgium, \\ \{quan.phan, gerda.janssens\}@cs.kuleuven. be
}

\begin{abstract}
Region-based memory management is a form of compiletime memory management, well-known from the functional programming world. This paper describes region-based memory management for the Mercury language using some points-to graphs that model the partition of the memory used by a program into separate regions and distribute the values of the program's variables over the regions. First, a region analysis determines the different regions in the program. Second, the liveness of the regions is computed. Finally, a program transformation adds region annotations to the program for region support. Our approach obtains good results for a restricted set of deterministic Mercury programs and is a valid starting point to make region-based memory management work with general Mercury programs.
\end{abstract}

\section{Introduction and Motivation}

Logic programming (LP) languages aim to free programmers from procedural details such as memory management tasks. One classical, automatic memory management technique in logic programming is to use a heap memory for structured terms and rely on backtracking and runtime garbage collection to reclaim memory. While efficient implementations of garbage collectors for LP languages can reuse more than $90 \%$ of the heap space of a program, they incur execution overhead because collectors often need to temporarily stop the main program.

To remedy this shortcoming compile-time techniques automatically enhance programs with instructions to reuse memory. This static method generally follows two approaches: region-based memory management (RBMM) and compiletime garbage collection (CTGC). The basic idea of RBMM is to divide the heap memory used by a program into different regions. The dynamically created terms and their subterms have to be distributed over the regions in such a way that at a certain point in the execution of the program all terms in a region are dead and the region can be removed. RBMM is a topic of intensive research for functional programming languages $[13,1,4,12]$ and more recently also for imperative languages [3, 2]. For LP languages, there has been only one attempt to make RBMM work for Prolog $[7,6,5]$. CTGC detects for a program when allocated

\footnotetext{
* This work is supported by the project GOA/2003/08 and by FWO Vlaanderen.
} 
memory cells are no longer used and instructs the program to reuse those cells for constructing new terms, reducing the memory footprint and in some cases achieving faster code. This idea has been used to reuse memory cells locally in the procedures of Mercury programs [9, 10, 8].

Our ultimate research goal is to investigate the possibility and practicality of a hybrid memory management technique, which combines RBMM and CTGC to capitalize on their advantages. As CTGC was developed for Mercury, relying much on Mercury-specific information such as determinism, modes, and types, which are not readily available in other LP systems it is a logical choice to develop RBMM in the same context. Moreover, this information can also be exploited in our model of the heap when it is divided into regions and in the region analyses.

The contribution of this paper is to develop an automated system based on program analysis that adds region annotations to Mercury programs. While program analyses based on points-to graphs are popular for languages with destructive update, in LP our work is the first to use the points-to graph to model the heap and to develop the program analyses that derive the model and make use of it for memory management.

In Section 2 we explain how memory management for Mercury can be based on the use of regions. The whole algorithm is composed of three phases, which are described in Sections 3, 4, and 5, respectively. Section 3 introduces the concept of the region points-to graph and the points-to analysis. Section 4 defines the live region analysis which uses the region points-to graph of each procedure to precisely detect the lifetime of the regions. We do the transformation by adding RBMM annotations in Section 5. Section 6 discusses the results of our prototype analysis system. Finally, Section 7 concludes.

\section{Regions and Mercury}

\subsection{Mercury programs}

We assume that the input of our program analysis is a Mercury program that is transformed by the MMC into superhomogeneous form, with the goals reordered and each unification specialized into a construction $(<=)$, a deconstruction $(=>)$, an assignment $(:=)$, or a test $(==)$ based on the modes [11]. The qsort program then consists of the following procedures (Fig. 1) ${ }^{1}$ :

\section{$2.2 \quad$ Usage of Regions: An Example}

We illustrate the usefulness of distributing terms over regions using the qsort example. In the implementation of LP languages, a list consists of the list skeleton and the elements. Observing the memory behaviour of the qsort procedure, we

\footnotetext{
1 The bold text can be ignored as it is the region annotations added automatically by the transformation. The italic comments are the corresponding transformation rules, which are described later in Sect. 5.
} 


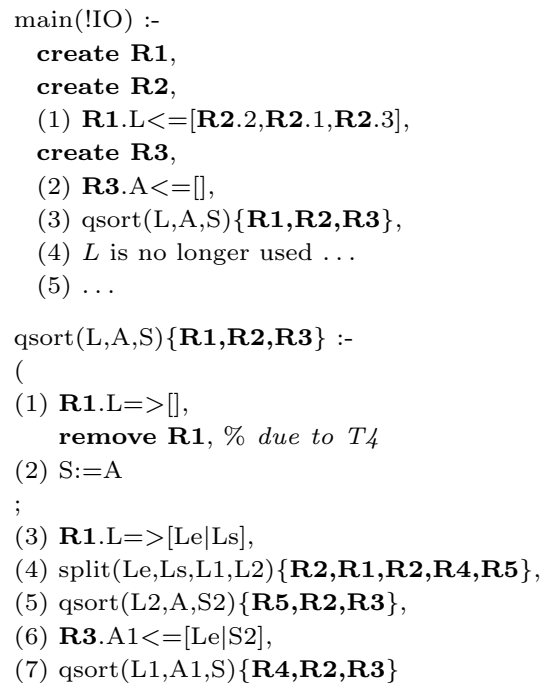

Fig. 1. qsort program.

see that the output list has a new skeleton built up in the accumulator while its elements are those of the input list. In the main predicate, the input list $L$ is no longer used after the call to qsort. This means that if the skeleton of the input list, the elements, and the skeleton of the output list (and the accumulator) are stored in three different regions we can safely free the memory occupied by the input list's skeleton by removing its region after the call. Take a closer look inside the qsort procedure at (4). The call to split creates two new lists with two new skeletons while the elements are also those of the input list. Therefore, if the two new skeletons are stored in regions different from the region of the input list's skeleton, the region can even be removed after this call inside qsort. This means that we detect even an earlier point where the region can be removed. So, by storing different components of the lists in separate regions we can do timely removal, recovering dead memory sooner.

The qsort program with region support produced by our analysis is shown in Fig. 1 with $\left\{\ldots, R_{i}, \ldots\right\}$ the list of region parameters of a procedure. Two instructions create and remove manipulate the creation and removal of regions. By R.X we denote the variable $X$ and its region. Detailed information about the regions for the variables will be given in Fig. 3. In qsort procedure, the region of the skeleton of the list $L$ passed to qsort from main is removed in the base case branch of split in the call at (4). The two new skeletons of the lists L1 and L2 are allocated in two separate regions, which are created in the base case branch of split. Those regions are removed in the calls at (5) and (7). If $L 1$ and $L 2$ are empty lists their regions are removed in the base case branch of qsort. Otherwise they are removed in the base case branch of split the same as what happens to the input list $L$. The region of the output list's skeleton is the region of the accumulator, which is created in main. 


\section{3 $\quad$ Storing Terms in Regions}

The way terms are allocated in memory depends on the specific implementation of a language. Therefore, we discuss the term representation when the heap memory is divided into regions in the Melbourne Mercury Compiler (MMC).

In the MMC, the way a term is stored depends on its type. Terms can be either of primitive types such as integer, char, float and string, or of discriminated union types. The latter types whose type constructors are functors with arity zero are called enumeration types, otherwise they are called record types. Primitive and enumeration terms are stored in a single memory cell. A term of a record type is stored on the heap and pointed to by a tagged pointer where the tag encodes the principal functor of the term and the pointer itself points to the block of memory cells corresponding to the arguments (subterms) of the principal functor. Fig. 2(a) shows the representation of a primitive type variable $H$ bound to an integer and of a record type variable $L$ bound to a list of two integers. A box with a slim border is a location on the stack or registers, one with a bold border is a location on the heap.

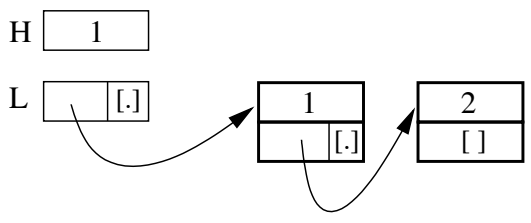

(a) In the MMC

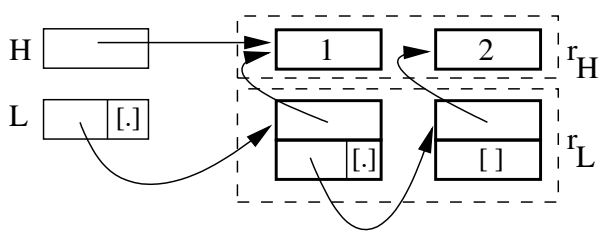

(b) In RBMM

Fig. 2. $\mathrm{H}=1, \mathrm{~L}=[\mathrm{H}, 2]$.

Now we consider the term representation when the heap is split into regions. To simplify the presentation in this paper, we assume that all terms are constructed on the heap and that the subterms of a compound term are always stored as if they are of record type. A primitive or enumeration term is stored in a memory cell in a region. To store a compound term, a region is used to store the memory block of its subterms. For a variable that is bound to the term, this region is called the region of the variable. If a subterm is of the same type as its compound term, it is stored in the same region as the compound term. Otherwise it is stored in a different region. Fig. 2(b) shows the division of the heap into regions by using dashed lines. The variable $H$ is bound to an integer that is stored in the region $r_{H}$. The two-cell memory blocks making up the skeleton of the list to which $L$ is bound are put into the other region, called the region of $L$ $\left(r_{L}\right)$, because the second subterm of a list is also of type list. Also, the elements reachable from the skeleton in $r_{L}$ are put in the same region, here $r_{H}$.

There are several good reasons for our choice. First, the representation ensures that the terms of a recursive type are always stored in a finite number of 
physical regions, regardless of the terms' actual sizes. Second, a program often treats the values of the same type in a term in the same way. By putting those values into the same region we can hopefully remove the region when the program no longer needs that part of the term, while other parts are still needed. Alternatively, the term bound to a variable could be stored entirely in a region, which is not a good approach when only a part of the term becomes dead. Another finer-grained approach could be that every subterm of a term is stored in a separate region. This approach would mean that the number of physical regions needed to store a recursive term depends on the size of the term (which usually is not known at compile-time). Those regions could not be known at compile-time therefore it would be difficult to keep track and manipulate them.

\section{Region Points-to Analysis}

The goal of this analysis is to build, for each procedure, a region points-to graph that represents the partition of the memory used by the procedure into regions. The concept of a region points-to graph was introduced for Java in [2] and we adapted it in the context of Mercury. For a procedure $p$, a region points-to graph, $G=(N, E)$, consists of a set of nodes, $N$, representing regions and a set of directed edges, $E$, representing references between the regions. A node has an associated set of variables of $p$ which are stored in the region corresponding to the node ${ }^{2}$. For a node $n$, its set of variables is denoted by $\operatorname{vars}(n)$. The node $n_{X}$ denotes the node such that $X \in \operatorname{vars}\left(n_{X}\right)$. A directed edge is a triple. $(m,(f, i), n)$ denotes an edge between $m$ and $n$, which is labelled by the type selector $(f, i)$ and represents the structured relation between the variables in the two nodes. The type selector $(f, i)$ selects the $i^{\text {th }}$ argument of the functor $f[8]$.

The points-to graphs of split and qsort procedures are shown in Fig. 3. For split we see that the skeletons of the lists $L$ and $L s$ are in the same region and that the elements are in the region pointed to by the edge with a label ([.],1). Note that the edge with label a ([.], 2) is for the skeleton.

The region points-to graph, $G=(N, E)$, of a procedure $p$ has to ensure the following invariants.

1. If a unification $X=f\left(\ldots, X_{i}, \ldots\right)$ (i.e., $<=$ or $=>$ ) appears in $p$ then $n_{X}, n_{X_{i}} \in N$ and there exists one and only one edge with a label $(f, i)$ from $n_{X}$ and $\left(n_{X},(f, i), n_{X_{i}}\right) \in E$.

2. Every variable of $p$ belongs to exactly one node and the variables in a node have the same type, which is regarded as the type of the node.

The task of the region points-to analysis then is to produce a graph that satisfies the invariants for each procedure in a program. The region points-to analysis is flow-insensitive, i.e., the execution order of the literals in a procedure does not matter, and consists of two analyses. One is an intraprocedural analysis that only deals with specialized unifications, ignoring procedure calls and the

\footnotetext{
${ }^{2}$ From now on, we use the concepts of a node and the region corresponding to the node interchangeably.
} 


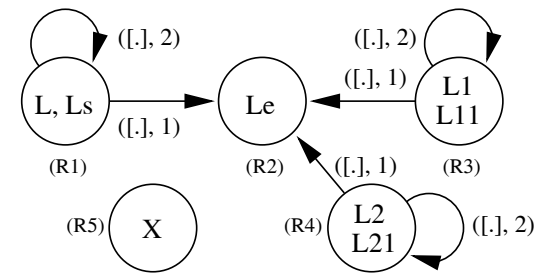

(a) split

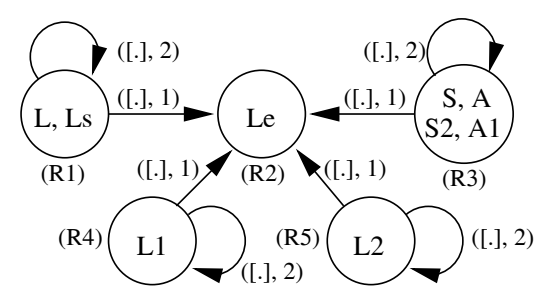

(b) qsort

Fig. 3. The points-to graphs of split and qsort.

other is an interprocedural analysis that integrates the points-to graph of the called procedure (callee) with that of the calling procedure (caller) at each call site. The interprocedural analysis requires a fixpoint computation to calculate points-to graphs for recursive procedures. The points-to analysis applies the operations unify and edge to $G=(N, E)$ and updates $G$ as follows.

- unify $(n, m)$ : unify nodes $n$ and $m$ in the graph.

- $N \leftarrow N \backslash\{n, m\} \cup\{k\}, k$ is a new node and $\operatorname{vars}(k)=\operatorname{vars}(n) \cup \operatorname{vars}(m)$.

- $E \leftarrow E$ with the edges where all appearances of $m, n$ are replaced by $k$.

- edge $(n, s e l, m)$ : create an edge with a label sel from node $n$ to node $m$.

- $G \leftarrow(N, E \cup\{(n, s e l, m)\})$.

\subsection{Intraprocedural Analysis}

To specify this analysis, assume that we are analysing a procedure $p$ with pointsto graph $G=(N, E)$. The analysis works as follows.

1. Each variable in $p$ is assigned to a separate node: for a variable $X, n_{X}$ becomes a node in $N$ and $\operatorname{vars}\left(n_{X}\right)=\{X\}$.

2. The specialized unifications in $p$ are processed one by one as follows:

- An assignment $X:=Y$ : record that $X$ and $Y$ are in the same region because they point to the same memory block, i.e., unify $\left(n_{X}, n_{Y}\right)$.

- A test $X==Y$ : do nothing.

- A deconstruction $X=>f\left(X_{1}, \ldots, X_{n}\right)$ or a construction $X<=f\left(X_{1}, \ldots, X_{n}\right)$ : create the references from $n_{X}$ to each of $n_{X_{1}}, \ldots, n_{X_{n}}$ by adding the edges edge $\left(n_{X},(f, 1), n_{X_{1}}\right), \ldots$, edge $\left(n_{X},(f, n), n_{X_{n}}\right)$.

3. The rules in Fig. 4 are fired whenever applicable. Rules $P 1$ and $P 2$ are to ensure the first invariant. Rule $P 3$ enforces the term representation for the variables of recursive types.

\subsection{Interprocedural Analysis}

The interprocedural analysis updates the region points-to graph of a procedure $p$ by integrating the relevant parts of the points-to graphs of the called procedures into it. Assume that for a call $q\left(Y_{1}, \ldots, Y_{n}\right)$, the head of the defining procedure is $q\left(X_{1}, \ldots, X_{n}\right)$. The analysis is performed as follows. 


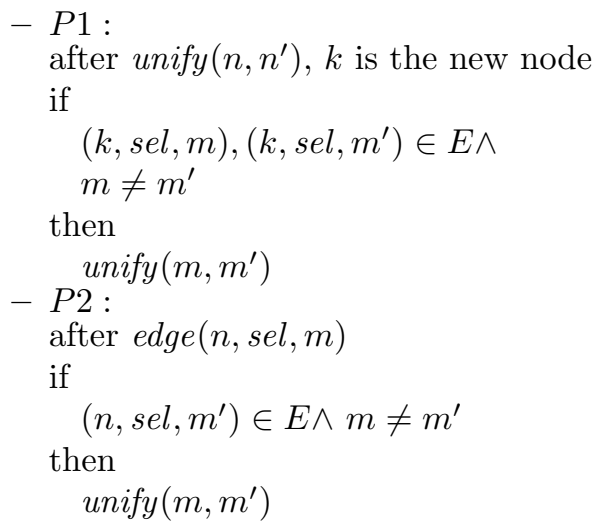

$-P 3:$

after $\operatorname{edge}(n, \operatorname{sel}, m)$

if

$\left(k_{1}, m\right) \in E^{+} \wedge$

$\left(m, k_{2}\right) \in E^{+} \wedge$

$k_{1} \neq k_{2} \wedge$

type $\left(k_{1}\right)=$ type $\left(k_{2}\right)$

then

$\operatorname{unify}\left(k_{1}, k_{2}\right)$

in which $E^{+}$is the reflexive, transitive closure of $E$ and type $(n)$ returns the type of the node $n$.

Fig. 4. Intraprocedural analysis rules.

1. Process each procedure call $q\left(Y_{1}, \ldots, Y_{n}\right)$ in $p$ : integrate the graph of $q$, $G_{q}=\left(N_{q}, E_{q}\right)$, into the graph of $p, G_{p}=\left(N_{p}, E_{p}\right)$ by building the partial $\alpha$ mapping from $N_{q}$ to $N_{p}$ as follows:

(a) Initializing the $\alpha$ mapping with $\alpha\left(n_{X_{1}}\right)=n_{Y_{1}}, \ldots, \alpha\left(n_{X_{n}}\right)=n_{Y_{n}}$. For those nodes $n_{X_{i}}$ 's that have been unified in $G_{q}$, the corresponding nodes $n_{Y_{i}}$ 's in the $G_{p}$ are also unified. This is achieved by applying rule $P 4$ in Fig. 5 to ensure that $\alpha$ is a function.

(b) In the graph $G_{q}$, start from each $n_{X_{i}}$, follow each edge once and apply rules $P 5-P 8$ in Fig. 5 when applicable. Those rules are to complete the $\alpha$ mapping and to copy the parts of $G_{q}$ that are relevant to $n_{X_{i}}$ 's into $G_{p}$. After some two nodes of $G_{p}$ are unified (rules $P 4$ and $P 5$ ) or an edge is added to $G_{p}$ (rules $P 7$ and $P 8$ ) we need to apply rules $P 1$ or $P 3$ ( $P 2$ is not applicable because its conditions cannot be satisfied) respectively to $G_{p}$ in order to maintain the invariant that there is only one edge with a given selector between two nodes and to conform with the term representation for recursive types.

2. Step 1 is repeated until there is no change in $G_{p}$.

For the qsort example, the region points-to graphs of split and qsort after the points-to analysis reaches a fixpoint is exactly as shown in Fig. 3.

\section{Live Region Analysis}

The goal of live region analysis is to detect live regions at each program point and to decide which regions are created and removed by each procedure.

With every literal in the body of a procedure $p$, a program point is associated. An execution path in $p$ is a sequence of program points, such that at runtime the literals associated with these program points are performed in sequence. We use the notions before and after a program point. Before a program point means the associated literal has not been executed, while after a program point means when its literal has just completed. The set of live regions (LR) at a program point is computed via the set of live variables (LV) at the program point. 


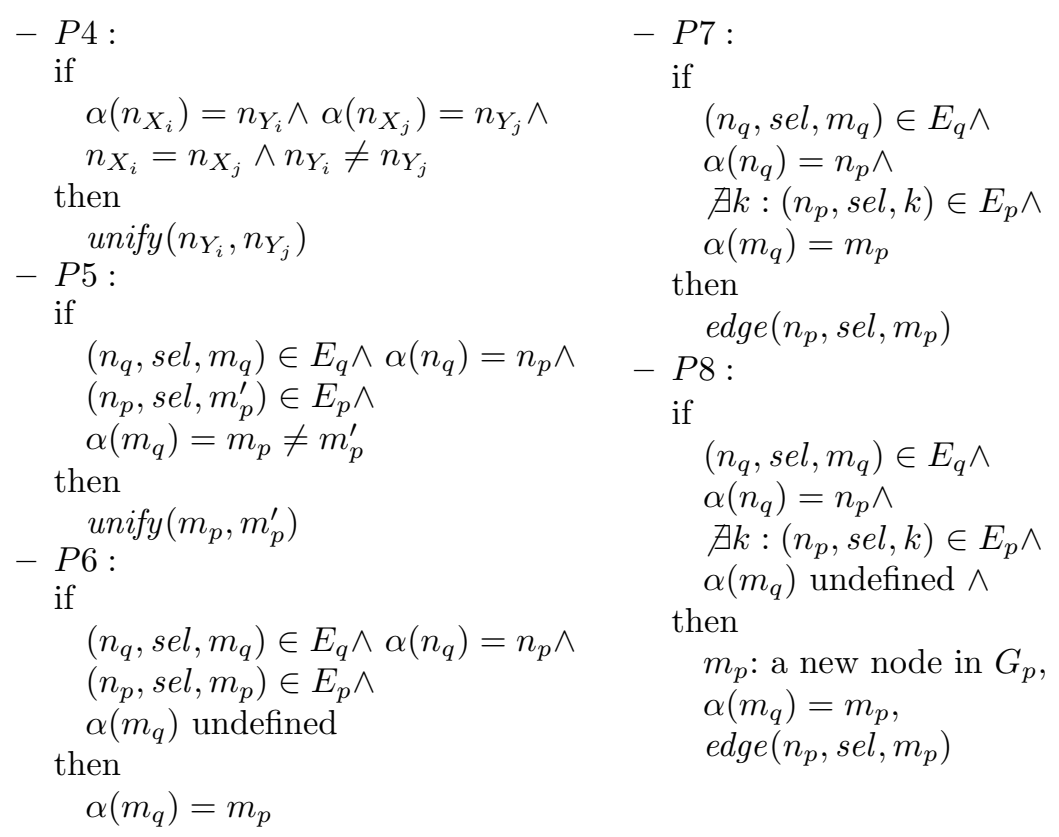

Fig. 5. Interprocedural analysis rules.

\subsection{Live Variables at a Program Point}

A variable is live after a program point in $p$ if:

- There exists an execution path in $p$ containing the program point that instantiates the variable before or at the program point and uses it after the program point,

- OR it is an output variable of $p$, which is instantiated before or at the program point.

If we call pre_inst $(i, P)$ the set of variables instantiated before the program point $i$ in the execution path $P$, post_use $(i, P)$ the set of variables used after $i$ in $P$, out $(i)$ the set of variables instantiated by the goal at $i$, out $(p)$ the set of output variables of a procedure $p$ then the set of live variables after $i$ is:

$L V_{\text {after }}(i)=\{V \mid \exists P: V \in($ pre_inst $(i, P) \cup$ out $(i)) \cap($ out $(p) \cup$ post_use $(i, P))\}$.

If we call $i n(i)$ the set of input variables to the literal at $i$, the set of live variables before $i$ is:

$$
L V_{\text {before }}(i)=\left(L V_{\text {after }}(i) \backslash \text { out }(i)\right) \cup \text { in }(i) .
$$

The $L V_{\text {before }}$ of the first program point of an execution path of a procedure $p$ is defined to be in $(p)$, the set of input variables of the procedure. The $L V_{\text {after }}$ of the last program point is defined to be out $(p)$. 


\subsection{Live Regions at a Program Point}

A region is live at a program point if it is reachable from a live variable at the program point. The set of regions that are reachable from a variable is defined:

$$
\operatorname{Reach}(X)=\left\{n_{X}\right\} \cup\left\{m \mid \exists\left(n_{X}, m\right) \in E^{*}(X)\right\},
$$

in which $E^{*}(X)$ is defined:

$$
E^{*}(X)=\left\{\left(n_{X}, n_{i}\right) \mid \exists\left(n_{X}, \text { sel }_{0}, n_{1}\right), \ldots,\left(n_{i-1}, \text { sel }_{i-1}, n_{i}\right) \in E\right\} .
$$

The LR sets before and after a program point $i$ are defined:

$$
\begin{aligned}
L R_{\text {before }}(i) & =\bigcup(\operatorname{Reach}(X)) \forall X \in L V_{\text {before }}(i) . \\
L R_{\text {after }}(i) & =\bigcup(\operatorname{Reach}(X)) \forall X \in L V_{\text {after }}(i) .
\end{aligned}
$$

\subsection{The Analysis}

This analysis computes for each procedure the LR sets before and after each program point and the set of regions that the procedure may create, called born $R$ and the set of regions that it may remove, called deadR.

First, LR sets can be computed in two passes. The first pass is a syntax-based analysis to compute the LV sets for each program point. Note that because of mode correctness the LV sets are specific for a program point, independent of execution paths containing it. The second pass computes the LR sets for each program point from the corresponding LV sets and the region points-to graph.

After that, to reason about the lifetime of regions across procedure boundary, for a procedure $p$ with its region points-to graph $G=(N, E)$, we define:

- $\operatorname{inputR}(p)$ is the set of regions reachable from input variables.

- outputR $(p)$ is the set of regions reachable from output variables.

- $\operatorname{born} R(p)$ is the set of output regions that the procedure or any of the procedures it calls may create. Initially born $R(p)=\operatorname{output} R(p) \backslash \operatorname{input} R(p)$.

- $\operatorname{deadR}(p)$ is the set of input regions that the procedure or any of the procedures it calls may remove. Initially $\operatorname{dead} R(p)=\operatorname{input} R(p) \backslash$ output $R(p)$.

- $\operatorname{localR}(p)=N \backslash \operatorname{inputR}(p) \backslash$ outputR $(p)$.

The analysis then follows each execution path of a procedure $p$ and applies the rules in Fig. 6 to any call $q$ to update the deadR and born $R$ sets of $q$. Essentially, a region is removed from $\operatorname{dead} R(q)$ if it needs to be live after the call to $q$ in $p$; or if it is going to be removed more than once by $q$. A region is removed from $\operatorname{born} R(q)$ if the region is already live before the call to $q$; or if $q$ is going to create the region more than once. When there is a change to those sets of $q, q$ needs to be analysed to propagate the change to its called procedures. Therefore, this analysis requires a fixpoint computation.

In the qsort program, split has three execution paths $\langle(1),(2),(3)\rangle,\langle(4),(5),(6),(7)\rangle$, and $\langle(4),(8),(9)\rangle$; qsort has two execution paths $\langle(1),(2)\rangle$ and $\langle(3),(4),(5),(6),(7)\rangle$. The LV and LR sets of split are in Tab. 1, of qsort in Tab. 2. Their corresponding deadR and born $R$ sets are: deadR(split $)=\{R 1\}$, born $R($ split $)=\{R 3, R 4\}$; $\operatorname{dead} R($ qsort $)=\{R 1\}, \operatorname{born} R($ qsort $)=\phi$. 


$$
\begin{aligned}
& -L 1: \quad-L 3: \\
& \text { if } \\
& r \in L_{\text {before }}(p p(l)) \wedge \\
& r \in L_{\text {after }}(p p(l)) \wedge \\
& r=\alpha\left(r^{\prime}\right) \wedge r^{\prime} \in \operatorname{deadR}(q) \\
& \text { then } \\
& \operatorname{deadR}(q)=\operatorname{deadR}(q) \backslash\left\{r^{\prime}\right\} \\
& \text { if } \\
& r \in L R_{\text {before }}(p p(l)) \wedge \\
& r=\alpha\left(r^{\prime}\right) \wedge \\
& r^{\prime} \in \operatorname{bornR}(q) \\
& \text { then } \\
& \operatorname{bornR}(q)=\operatorname{bornR}(q) \backslash\left\{r^{\prime}\right\} \\
& \text { - L2: } \\
& \alpha\left(r^{\prime}\right)=r \wedge \alpha\left(r^{\prime \prime}\right)=r \wedge \\
& r^{\prime} \neq r^{\prime \prime} \wedge \\
& r^{\prime} \in \operatorname{deadR}(q) \\
& \operatorname{deadR}(q)=\operatorname{deadR}(q) \backslash\left\{r^{\prime}\right\} \\
& \text { - L4: } \\
& \operatorname{bornR}(q)=\operatorname{bornR}(q) \backslash\left\{r^{\prime}\right\}
\end{aligned}
$$

Fig. 6. Live region analysis rules.

\section{Program Transformation}

The goal of program transformation is to introduce create and remove instructions based on the region liveness information. Each procedure is transformed by following its execution paths and applying the transformation rules in Fig. 7 to each program point. Assuming that we are analysing a procedure $p$. Let $l_{i}$ be the associated literal at a program point $i$ in $p$. A literal can be either a specialized unification denoted by unif or a call (user-defined or built-ins). We assume that all the specialized unifications as well as the built-ins do not remove or create any regions.

\begin{tabular}{|c|c|c|c|c|}
\hline pp & $L V_{\text {before }}$ & $L V_{\text {after }}$ & $L R_{\text {before }}$ & LR after \\
\hline \hline$(1)$ & $\{X, L\}$ & \{\} & $\{R 5, R 1, R 2\}$ & \{\} \\
$(2)$ & \{\} & $\{L 1\}$ & \{\} & $\{R 3, R 2\}$ \\
$(3)$ & $\{L 1\}$ & $\{L 1, L 2\}$ & $\{R 3, R 2\}$ & $\{R 3, R 2, R 4\}$ \\
$(4)$ & $\{X, L\}$ & $\{X, L e, L s\}$ & $\{R 5, R 1, R 2\}$ & $\{R 5, R 2, R 1\}$ \\
$(5)$ & $\{X, L e, L s\}$ & $\{X, L e, L s\}$ & $\{R 5, R 2, R 1\}$ & $\{R 5, R 2, R 1\}$ \\
$(6)$ & $\{X, L e, L s\}$ & $\{L 2, L e, L 11\}$ & $\{R 5, R 2, R 1\}$ & $\{R 4, R 2, R 3\}$ \\
$(7)$ & $\{L 2, L e, L 11\}$ & $\{L 1, L 2\}$ & $\{R 4, R 2, R 3\}$ & $\{R 3, R 2, R 4\}$ \\
$(8)$ & $\{X, L e, L s\}$ & $\{L 1, L e, L 21\}$ & $\{R 5, R 2, R 1\}$ & $\{R 4, R 2, R 3\}$ \\
$(9)$ & $\{L 1, L e, L 21\}$ & $\{L 1, L 2\}$ & $\{R 4, R 2, R 3\}$ & $\{R 3, R 2, R 4\}$ \\
\hline
\end{tabular}

Table 1. Live variable and live region sets of split. 


\begin{tabular}{|c|c|c|c|c|}
\hline $\mathrm{pp}$ & $L V_{\text {before }}$ & $L V_{\text {after }}$ & LR before & $L_{\text {after }}$ \\
\hline \hline$(1)$ & $\{L, A\}$ & $\{A\}$ & $\{R 1, R 2, R 3\}$ & $\{R 3, R 2\}$ \\
$(2)$ & $\{A\}$ & $\{S\}$ & $\{R 3, R 2\}$ & $\{R 3, R 2\}$ \\
$(3)$ & $\{L, A\}$ & $\{A, L e, L s\}$ & $\{R 1, R 2, R 3\}$ & $\{R 3, R 2, R 1\}$ \\
$(4)$ & $\{A, L e, L s\}$ & $\{A, L e, L 1, L 2\}$ & $\{R 3, R 2, R 1\}$ & $\{R 3, R 2, R 4, R 5\}$ \\
$(5)$ & $\{A, L e, L 1, L 2\}$ & $\{L e, L 1, S 2\}$ & $\{R 3, R 2, R 4, R 5\}$ & $\{R 2, R 4, R 3\}$ \\
$(6)$ & $\{L e, L 1, S 2\}$ & $\{L 1, A 1\}$ & $\{R 2, R 4, R 3\}$ & $\{R 4, R 2, R 3\}$ \\
$(7)$ & $\{L 1, A 1\}$ & $\{S\}$ & $\{R 4, R 2, R 3\}$ & $\{R 3, R 2\}$ \\
\hline
\end{tabular}

Table 2. Live variable and live region sets of qsort.

A region is created when it first becomes live, namely when the region is not live before $i$ but is live after $i$. If $l_{i}$ creates the region ${ }^{3}$, then no annotation is added at $i$. Otherwise the region is created either by a caller of $p$ or by $p$ itself. The former means that the region does not need to be created again in $p$ and no annotation is added at $i$. The latter occurs when the region belongs to either $\operatorname{born} R(p)$ or $\operatorname{local} R(p)$ and a create instruction is added before $l_{i}$.

A region is removed when it ceases to be live. The first situation is when the region is live before $i$ but not live after $i$. If $l_{i}$ removes the region, then no remove instruction needs to be inserted in $p$. Otherwise if the region is removed by $p$ itself, namely the region is in either $\operatorname{dead} R$, localR, or born $R$ sets of $p$, a remove instruction is inserted after $l_{i}$ to remove the region. While the reason for removing the region when it belongs to the first two sets is straightforward, the removal of a region in $\operatorname{born} R(p)$ is allowed because it is acceptable for $p$ to remove the region after $i$ and re-create it later on (which $p$ will fulfill somehow because the region is in $\operatorname{born} R(p)$ ). If $p$ does not remove the region either then the region is removed by a caller of $p$ and no annotation is introduced at $i$. The second situation is when the region is live after $i$, but not live before some program point $j$ following $i$ in a certain execution path. This can happen when $i$ is a shared point among different execution paths and the region is live after $i$ due to an execution path to which $j$ does not belong. A remove instruction is added before $l_{j}$ to remove the region.

The result of the program transformation of the qsort program has been shown in Fig. 1.

\section{Prototype Implementation and Discussion}

We implemented a prototype of the algorithm as a source-to-source transformation in the MMC version 0.12.0. The analyser operates as one of the last analyses on the High Level Data Structure representation of the original source code and produces human-readable source code with region support. To realise Mercury with RBMM the runtime system of Mercury should be extended with support for regions, which is out of the scope of this paper.

\footnotetext{
${ }^{3}$ When we say a region is created (removed) by a procedure it means that the region is either created (removed) by the procedure itself or by one of the procedures that it calls.
} 


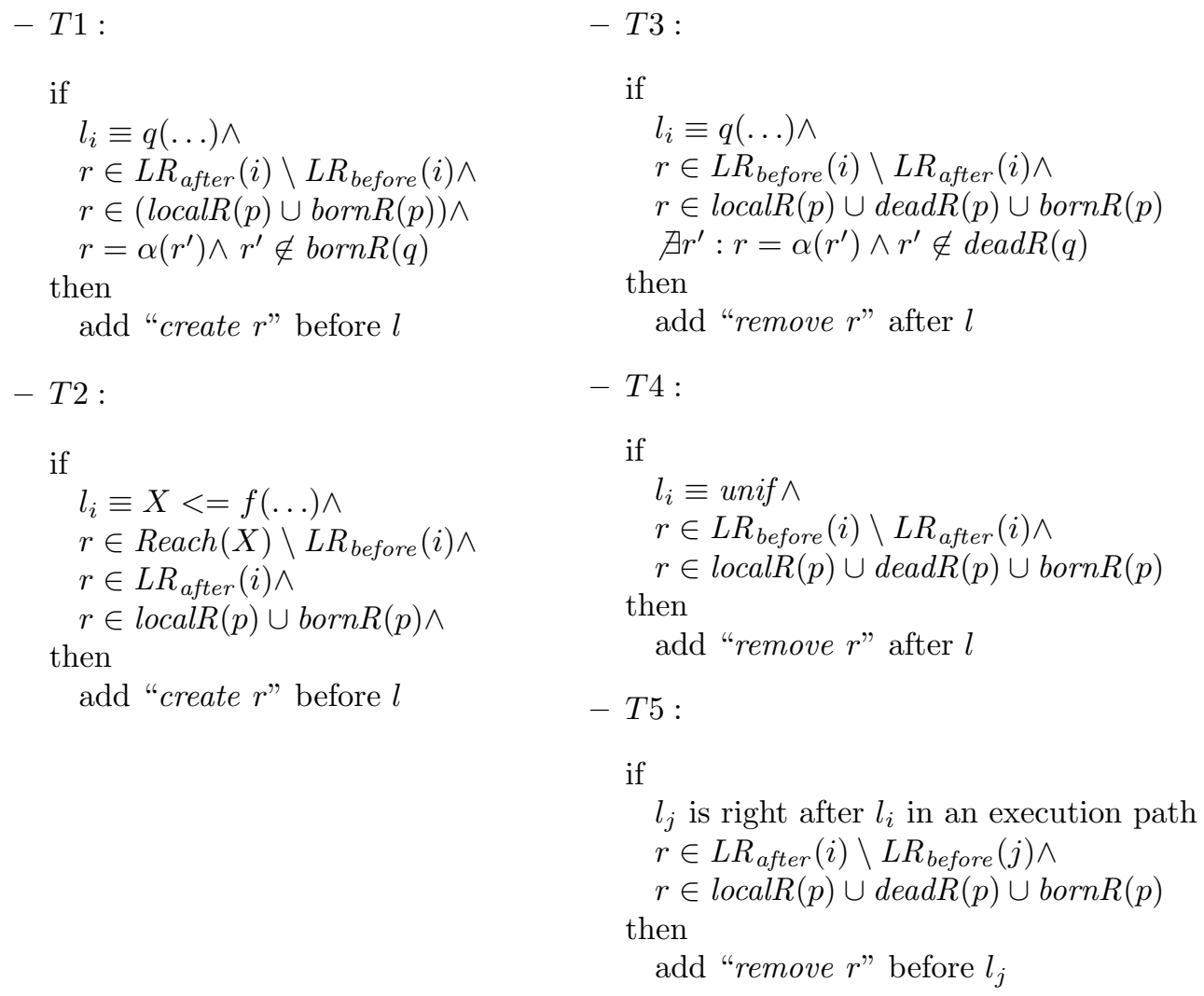

Fig. 7. Transformation rules.

Our region analysis and transformation work correctly for deterministic programs in which the condition of if-then-else constructs, if any, can only be a goal that does not require creating or removing any regions inside itself. Note that such a deterministic program can still have different execution paths. There are two reasons for these restrictions. Firstly, the region liveness analysis only takes into account forward execution. Therefore the removal and creation of regions are only correct w.r.t. forward execution as we have in deterministic programs. Secondly, even with a deterministic program, we need to be sure that when an execution path of the program is taken all the region instructions on that path are actually executed. A canfail goal could cause problems such as created regions would never be removed or a region would be created or removed more than once, and so on. In deterministic programs such canfail goals can only appear in the condition of if-then-elses. Therefore, the condition goal of if-then-elses is restricted so that no region instructions occur in this nondeterministic context.

Our approach is a valid starting point for RBMM for general Mercury programs. The authors in $[5,7]$ describe an enhanced runtime for Prolog with RBMM, which provide the support for nondeterministic goals. The idea of that 
enhanced runtime is that backtracking is made transparent to the algorithm for deterministic programs by providing a mechanism to undo changes to the heap memory, restoring the heap memory to the previous state at the point to which the program backtracks. We believe that with a similar runtime support our algorithm can be used unchanged to support full Mercury programs. In future work we will investigate whether the runtime support can be lessened by exploiting the determinism information available in Mercury.

To make it possible to study the programs with region support we implemented a region simulator in which create and remove instructions are valid Mercury predicates that record each time a region is created or removed. The simulator is not fully automated in the sense that a program annotated with the two predicates generated by our prototype needs to be manually modified so that it can be compiled and executed as a normal Mercury program. By using the simulator we collected the information about the total number of regions created and the maximum number of regions during a program execution. We can also verify that all regions created by the program are also removed when the program finishes. Unfortunately, the sizes of the regions, which are essential to measure memory saving, are not collected by the simulator. Collecting those figures as well as the runtime performance of the transformed programs would require a working system of Mercury with support for RBMM.

\begin{tabular}{|l|r|r|r|r|r|}
\hline & nrev & qsort & dnamatch & argo_cnters & life \\
\hline \hline Total number of regions & 500 & 1001 & 1448 & 2000 & 1216 \\
Maximum number of regions & 4 & 12 & 17 & 34 & 12 \\
Analysis time (sec) & 0.010 & 0.016 & 0.280 & 1.679 & 0.189 \\
Normal compilation time (sec) & 0.259 & 0.233 & 0.309 & 0.66 & 0.340 \\
\hline
\end{tabular}

Table 3. Experimental results.

The results in Tab. 3 show that the total number of regions is always much larger than the maximum number of regions. It means that there are quite many regions having a short lifetime and the programs are able to remove them. The analysis time ${ }^{4}$ ranges from $3.8 \%$ to $254 \%$ of the normal compilation time ${ }^{5}$, which is acceptable for a prototype implementation of the algorithm. The analysis time for argo_cnters is large likely due to the fact that this benchmark processes the functors of a large arity (20), creating a graph with many nodes and edges, and that the behaviour of the program itself causes many nodes to be unified.

Up to our knowledge, the pioneering and only attempt to apply RBMM to a standard LP system is the work of Makholm and Sagonas [7] for XSB Prolog. Their work focused on the WAM runtime extensions required for regions, not the region analysis. In that aspect, they did validate that RBMM can work

\footnotetext{
${ }^{4}$ The analyses are executed on a Pentium $42.8 \mathrm{MHz}$ with 512MB RAM running Debian GNU/Linux 3.1 machine, under a usual load.

${ }^{5}$ The compilation time using the MMC 0.12 .0 with default compilation options.
} 
well with nondeterministic code, a unique feature of logic programming. Our work has concentrated on the static analysis. In our experiment we use the benchmark programs nrev, qsort, and dnamatch, also used in [7]. Our algorithm produces better analysis results for the first two programs, as it distributes the list skeletons and the elements in different regions and detects a shorter lifetime of regions, which would imply less memory consumption at runtime. Our RBMM qsort and nrev programs use no more memory than what is needed to store the input list. While in [7] nrev used maximally double and qsort 1.66 times the memory size of the input list. For dnamatch our analysis has the same problem as reported in [7], when temporary data is put in the same regions as the input and output data. This problem also happens for agro_cnters and life, which should cause poor performance of RBMM.

In our analysis, the problem is due to the imprecision of the region pointsto analysis. The current region points-to analysis cannot capture the fact that two regions are the same in one execution path but not in another and just always unifies them. If they would have been kept separate, one of them had been removed when only the other is live. This problem has also been reported in the RBMM research for functional programming $[6,4]$ with many solutions such as storage mode analysis, and those in [1,4] but as far as we understand those solutions have not solved the problem completely. A tentative approach to tackle this problem is to enhance the region points-to analysis so that it is able to detect which variables are definitely in the same regions and which ones are possibly in the same regions. We will investigate this possibility in future work.

\section{Conclusion}

We have developed and implemented an algorithm for region analysis and transformation for deterministic Mercury programs. While the work in [7] emphasised on the extension of the Prolog runtime system for supporting regions, our work here focuses on the static detection of regions and the introducing region annotations, exploiting the available type and mode information of Mercury. Experimental results of using a prototype implementation of the algorithm are reported for several programs. We obtained promising results for some programs, while for some others the typical shortcomings known from the functional programming world show up. In particular, we now have a system in which the combination of CTGC and RBMM can be investigated. The correctness proofs of the analysis and transformation will be topics for further work. Future work also includes the modular region analysis and the improvement of the precision of the region points-to graph and analysis.

\section{References}

[1] A. Aiken, M. Fähndrich, and R. Levien. Better static memory management: Improving region-based analysis of higher-order languages. In Proceedings of the ACM SIGPLAN 1995 Conference on Programming Language Design and Implementation, pages 174-185. ACM Press, 1995. 
[2] S. Cherem and R. Rugina. Region analysis and transformation for Java. In Proceedings of the 4th International Symposium on Memory Management, pages 85-96. ACM Press., October 2004.

[3] D. Grossman, G. Morrisett, T. Jim, M. Hicks, Y. Wang, and J. Cheney. Regionbased memory management in Cyclone. In Proceedings of the ACM Conference on Programming Language Design and Implementation., pages 282-293. ACM Press., 2002.

[4] F. Henglein, H Makholm, and H. Niss. A direct approach to control-flow sensitive region-based memory management. In Principles and Practice of Declarative Programming., pages 175-186. ACM Press., 2001.

[5] H. Makholm. A region-based memory manager for Prolog. In Proceedings of the 2nd International Symposium on Memory Management, pages 25-34. ACM Press., 2000.

[6] H. Makholm. Region-based memory management in Prolog. Master's thesis, University of Copenhagen, 2000.

[7] H. Makholm and K. Sagonas. On enabling the WAM with region support. In Proceedings of the 18th International Conference on Logic Programming. Springer Verlag., 2002.

[8] N. Mazur. Compile-time garbage collection for the declarative language Mercury. $\mathrm{PhD}$ thesis, Department of Computer Science, Katholieke Universiteit Leuven, May 2004.

[9] N. Mazur, G. Janssens, and M. Bruynooghe. A module based analysis for memory reuse in Mercury. In Proceedings of Computational Logic - CL 2000, First International Conference, volume 1861 of Lecture Notes in Artificial Intelligence, pages 1255-1269. Springer-Verlag, 2000.

[10] N. Mazur, P. Ross, G. Janssens, and M. Bruynooghe. Practical aspects for a working compile time garbage collection system for Mercury. In Proceedings of the 17th International Conference on Logic Programming, volume 2237 of Lecture Notes in Computer Science, pages 105-119. Springer, 2001.

[11] Z. Somogyi, F. Henderson, and T. Conway. The execution algorithm of Mercury, an efficient purely declarative logic programming language. The Journal of Logic Programming, 29(1-3):17-64, October-December 1996.

[12] M. Tofte, L. Birkedal, M. Elsman, and N. Hallenberg. A retrospective on regionbased memory management. Higher-Order and Symbolic Computation, 17:245265,2004 .

[13] M. Tofte and J.-P. Talpin. Region-based memory management. Information and Computation., 132(2):109-176, February 1997. 